\title{
Perimeter Entropy and its Application to Climate Change
}

\author{
HONGXING CAO $^{1}$ JIE ZHOU ${ }^{2}$ JIAN SONG $^{3}$ \\ 1 Chinese Academy of Meteorological Sciences, Beijing, 100081 \\ CHINA. \\ 2 Chongqing Climate Center, Chongqing Province, \\ CHINA \\ 3 College of Sciences, Inner Mongolia University of Technology, Hohhot, 010051 \\ CHINA \\ caohx@sina.com; dmwsj@163.com
}

\begin{abstract}
Periphery phenomenon and its theory are introduced briefly; herein perimeter set is defined; and perimeter entropy is presented mathematically. By using precipitation and evaporation data over the middle and lower reaches of the Yangtze River, the perimeter entropy was computed. Analysis points out that with perimeter entropy study one can project climate change in advance.
\end{abstract}

Key-Words: Information Entropy Periphery Theory Climate Change Global Warming

Received: August 3, 2019. Revised: February 2, 2020. Accepted: February 19, 2020. Published: February 28, 2020.

\section{Introduction}

One can easily find the periphery(jieke 界壳 in Chinese) phenomena, for example, a hard shell of a turtle, a castle (Fig. 1), a livestock shed, watershed between two rivers, country or region boundary, firewall in the internet etc. But a package of goods is not a jieke, as they are out of exchange. A gang syndicate has an almost closed periphery, its members exchange less with other gangorganizations and society. Periphery theory is just to study the kind of periphery phenomena[1]; Nowadays periphery theory has been applied to many fields[2,3].

At first periphery phenomena and definition as well as periphery theory are briefed; herein perimeter set and perimeter entropy are presented mathematically. By using climate data over the middle and down reaches of Yangtze River the perimeter entropy has been calculated; and corresponding climate implication is analyzed. The paper is organized as follows: the periphery is described in section 2; periphery set is defined in section 3. In section 4, the positive, negative and total of entropy are given. Finally, in section 5, using precipitation and evaporation data over the middle and lower reaches of the Yangtze River, the perimeter entropy was computed, and conclusions are drawn.

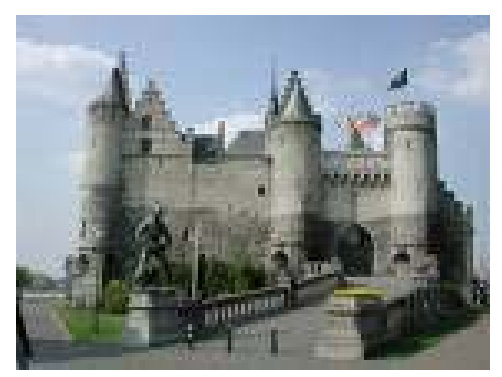

Fig.1. Castle

\section{Periphery Definition And Structure}

A periphery of the system is undoubtedly a part of the system, which situates on its boundary, and adjacent to environment. So the periphery is an intermediary agent between the system and its environment.

A kind of system boundary that plays a role in

-defending the existence of the system and

•exchanging between the system and its environment

is called as a periphery or jieke, Thereinafter both periphery and jieke will be used, depending on 
which is proper. The basic structure of a periphery(jieke) of system is composed of both a wall, which defends system itself, and a gate (or passage), through which the exchange between the system and its environment is carried out (Fig. 2).

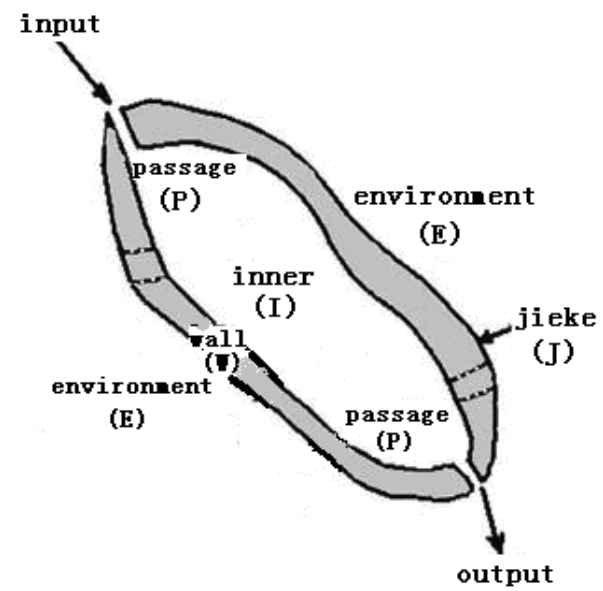

Fig.2. Schematic diagram of periphery

\section{Perimeter Set}

Suppose a periphery sub-unit in system boundary ( $\mathrm{SB}$ ) is $u$, which is a small part of system boundary, obviously there is

$$
S B=\bigcup_{i} u_{i}
$$

Defense degree $\mu(u)$ and exchange degree $v(u)$ of sub-unit are given,

$$
\mu(u), v(u) \in[0,1], \quad \mu(u)+v(u) \leq 1,
$$

Besides, the state $\zeta$ of the sub-unit is surely considered, the state change is related to the states of other parts of the system and affects to defensiveness and exchange rate.

So it needs to define a perimeter set [4]. Let us have a universe of discourse $E$, let $A$ be a subset of $E$. Then construct the set

$$
A=\left\{<u, \zeta_{A}(u), \mu_{A}(u), v_{A}(u)>\mid u \in E\right\},
$$

where $\zeta_{A}(u), \mu_{A}(u), v_{A}(u) \in[0,1]$, $\mu(u)+v(u) \leq 1$. We will call the set $A$ perimeter set with 3-logo. Suppose perimeter set with nelements, denote "+"expresses another sub-unit following, not algebra addition. For a continuous periphery with unlimited elements, denote

$$
A=\oint_{U}(\zeta, \mu, v) / u
$$

where notation $\oint$ is not a integral, but a perimeter set with unlimited elements. Denote system state $\zeta \in[0,1]$, then the sub-unit $u$ of periphery is expressed as

$z=(\zeta, \mu, v) / u$

But for the sub-unit $u$ of the system inner

$$
z=(\zeta, 0,0) / u
$$

Because the sub-unit $u$ of the system inner has no function of both defense and exchange, so $\mu=0, v=0$.

Define merging number

$$
\begin{aligned}
& M(u)=[\mu(u)+v(u) j]+\zeta(u) i=\kappa(u)+\zeta(u) i \\
& \mu(u)+v(u) j=\kappa(u)
\end{aligned}
$$

where $i$ is convert coefficient for system state to periphery elements: $\mu(u)+v(u), j$ is convert coefficient for the exchange degree $v(u)$ to the defense degree $\mu(u), \kappa(u)$ stands for total function of sub-unit $u$ of periphery. From Eq. (7) one knows the defense is a kernel of three logos. $M(u)$ is called a merging number. The merging number is similar to an imaginary number in form. The merging number is one of representative functions of the subunit of the periphery, but it contains the periphery state $\zeta$. Several ordinary mathematic methods, such as vector, 2-dimension diagram are able to analyze the merging number. Of course, $i$ and $j$ are required to determine by the observed data and specialist knowledge.

For example, the sub-unit of the periphery is just a gate, if the gate is close, then $\mu=1, v=0, \zeta=0$, taking $i=j=1, M(u)=1$; if the gate is open, then $\mu=0, v=1$, $\zeta=1$, taking $i=j=1, M(u)=2$; if the gate is half-close and half-open, then $\mu=0.5, v=0.5, \zeta=0.5$, taking $i=j=1, M(u)=1$.

$$
A=\left(\zeta_{1}, \mu_{1}, v_{1}\right) / \mu_{1}+\left(\zeta_{2}, \mu_{2}, v_{2}\right) / \mu_{2}+\cdots+\left(\zeta_{n}, \mu_{n}, v_{n}\right) / \mu_{n}^{i=j=1}
$$


For generality, defense and exchange are extended to positive and negative respectively in the following context.

\section{Entropy}

Suppose there are $n$ sub-unit of a system boundary, so correspondently there are

$$
\begin{gathered}
\mu_{1}, \mu_{2}, \cdots \mu_{n}, \mu(u) \in[0,1] \\
v_{1}, \nu_{2}, \cdots v_{n}, v(u) \in[0,1]
\end{gathered}
$$

Define positive entropy of a periphery

$$
H(\mu)=-\frac{1}{\ln n} \sum_{1}^{n} \mu_{i} \ln \mu_{i},
$$

Define negative entropy of a periphery

$$
H(v)=-\frac{1}{\ln n} \sum_{1}^{n} v_{i} \ln v_{i},
$$

Total entropy of periphery is

$$
H_{T}=H(\pi)+w H(\chi) .
$$

where $w$ is weight, dependent to practical issue studied.

\section{Case Computing And Conclusion}

Climate system is a thermo-dynamic one, which contains the atmosphere, the ocean, the land surface, biosphere and the cryosphere[5]. Once the elements of climate system are observed, the quantities of elements become the big data; people should process the data by use of information technology. So it is to say, study of climate system has to treat both physic-chemistry problem and information one. Herein we focus on information aspect of the climate system.

By using precipitation and evaporation data over the middle and lower reaches of the Yangtze River, the perimeter entropy was computed. For obtaining the data place between 0 and 1, the precipitation and evaporation data were pre-processed through

$z_{i}^{\prime}=\frac{z_{i}-z_{\text {min }}}{z_{\text {max }}-z_{\text {min }}}, i=1,2 \ldots m$.

where $z_{\min }, z_{\max }$ are minimum and maximum of $\mathrm{m}$ observations, $m$ is observed sample size. Regarding precipitation as positive quantity, but evaporation as negative one, then by use of Eq. (8), (9), perimeter entropies were calculated. There in Eq. (10), $w$ is taken as -1 , because for land surface the directions of precipitation and evaporation are opposite, the difference between precipitation entropy and evaporation one is given in Fig. 4. Fig. 5 displays the yearly temperature over the middle and lower reaches of the Yangtze River. Obviously, the difference trend decreases; but the temperature change increases, i.e. both exhibit opposite way and their correlation coefficient is negative. The temperature increase is part of global warming[6,7]. It is inferred that with perimeter entropy study one will possibly project climate change in advance. 

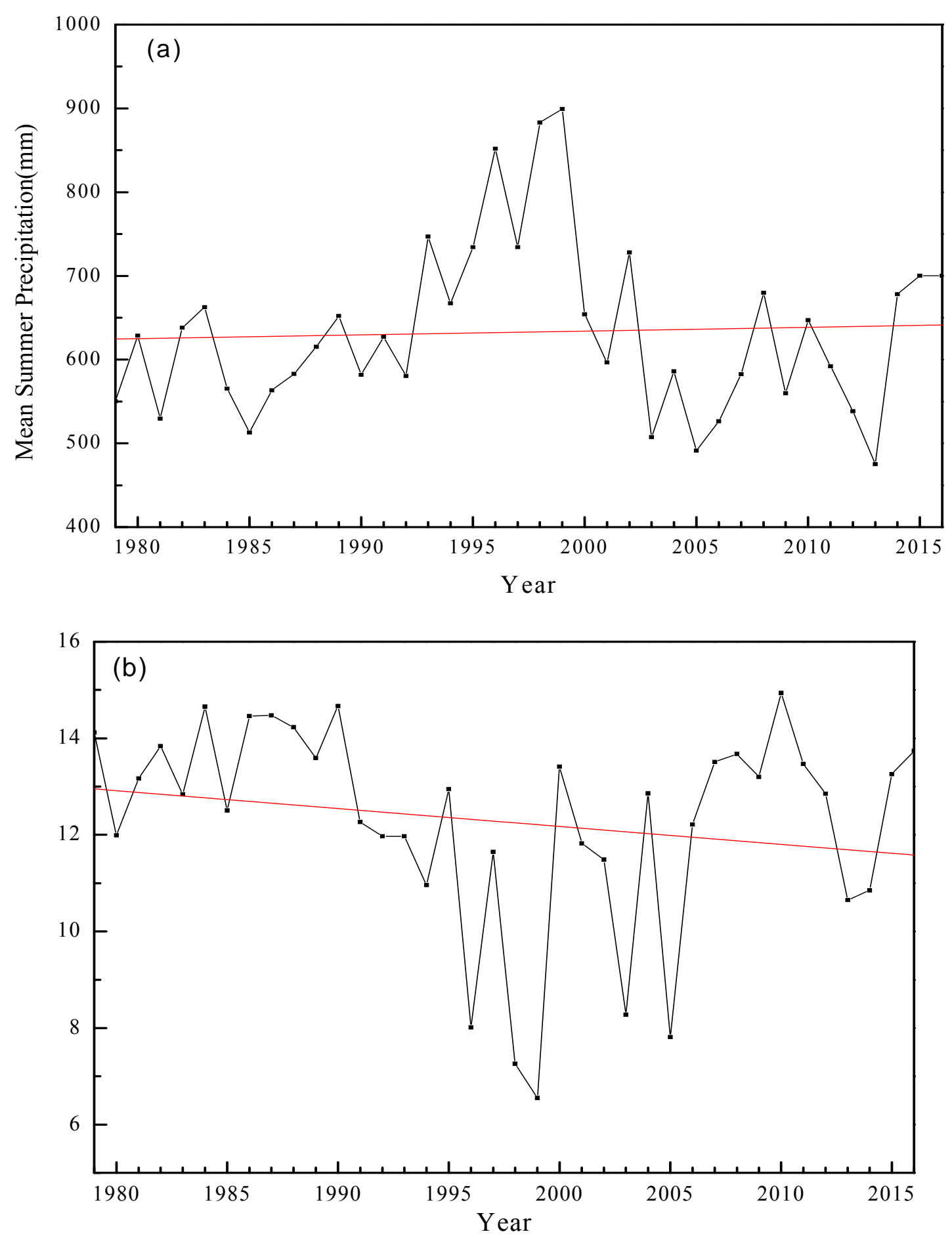

Fig.3. Yearly precipitation (a)and its perimeter entropy(b) over the middle and lower reaches of the Yangtze River(straight line: trend line from 1979 to 2015) 


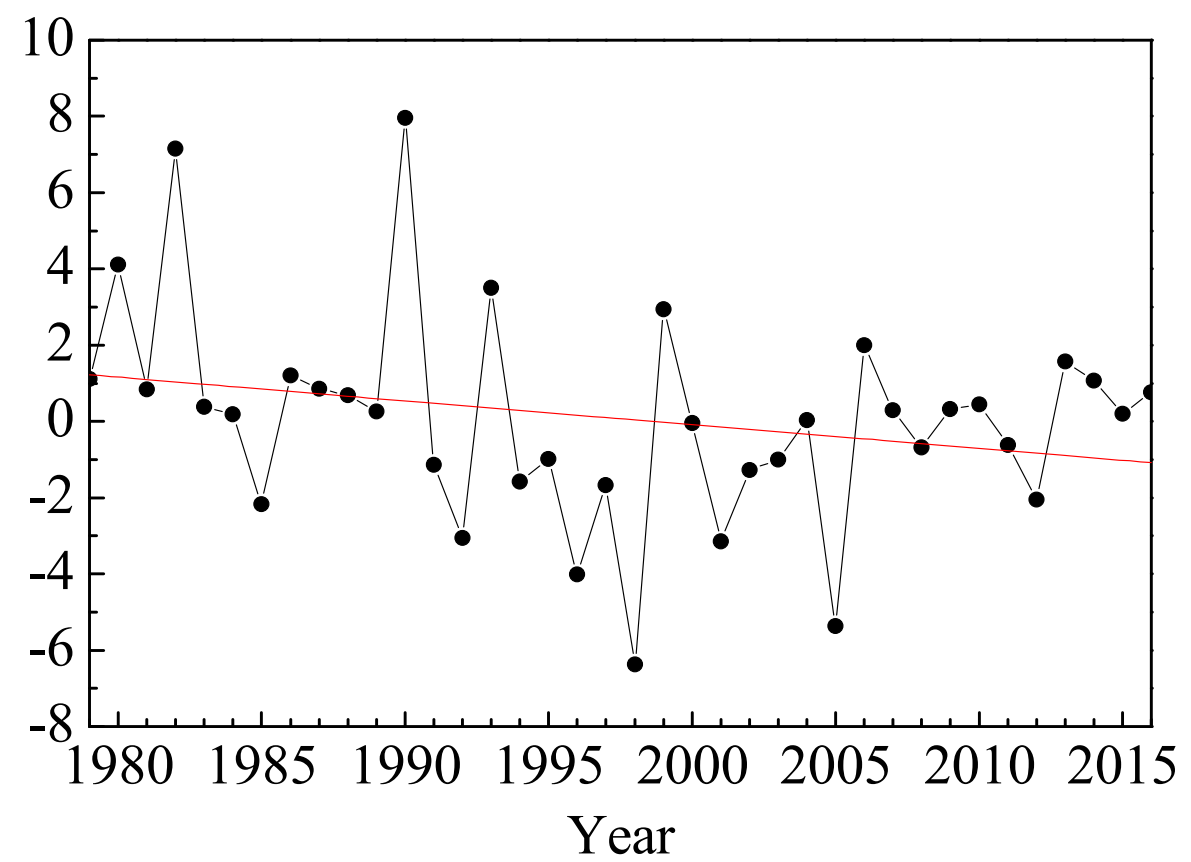

Fig.4. Difference between the perimeter entropy of precipitation and the perimeter entropy of evaporation (straight line: trend line from 1979 to 2016)

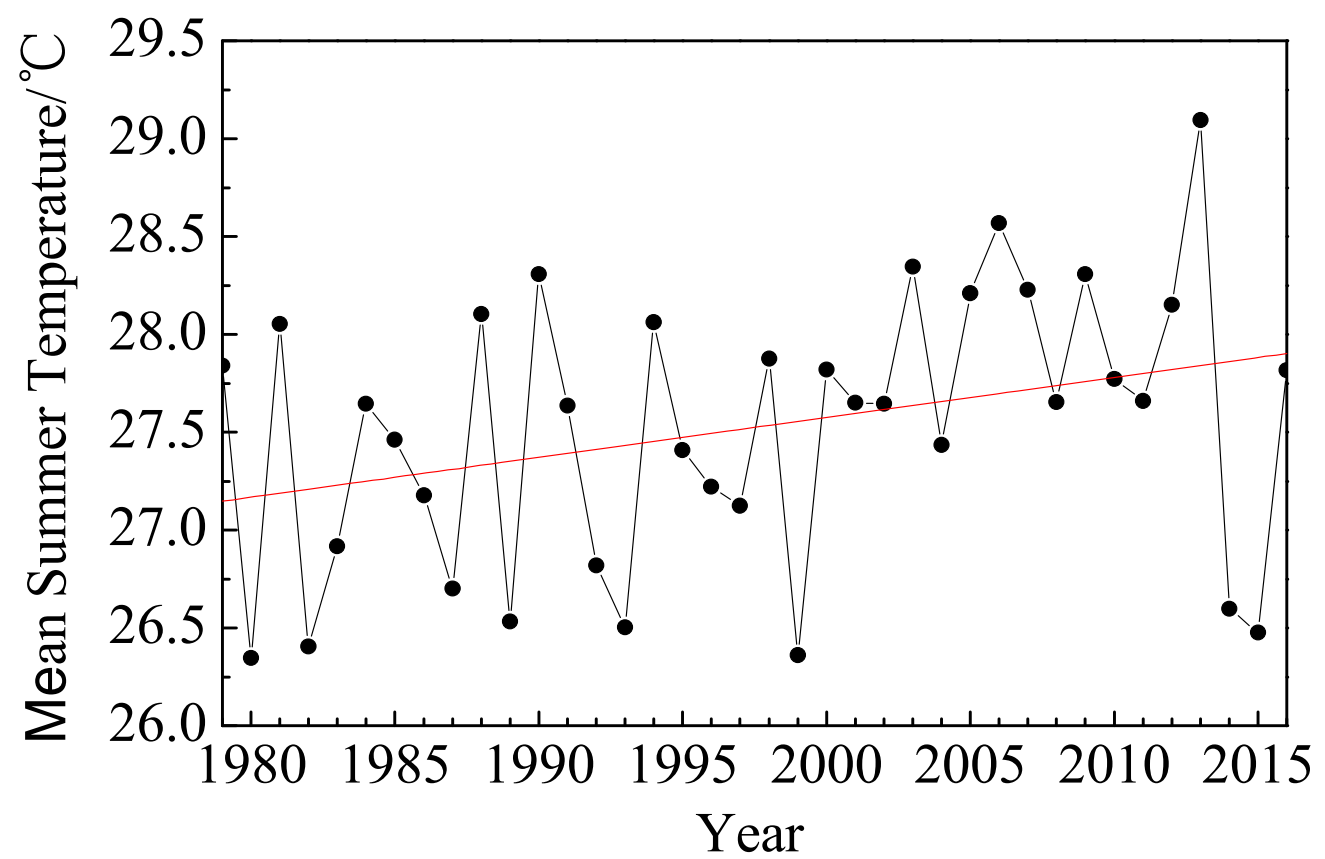

Figure.5. Yearly temperature variation of the middle and lower reaches of the Yangtze River (straight line: trend line from 1979 to 2016) 
Acknowledgment : The authors thank two anonymous reviewers. This work is supported by the National Natural Science Foundation of China, Grant No. 41375079 and Grant No. 41775092, the Natural Science Foundation of Inner Mongolia, Grant No. 2018LH04005. The original data sets were obtained from National Meteorological Information Center, http://data.cma.gov.cn .

\section{References:}

[1] Cao Hongxing, "Modelling of a system boundary," Kybernetes, vol. 24, no.6, pp44-49, 1995.

[2] Cao Y. Hongxing, Modeling of System Boundary-Periphery Theory. Staarbruecken, Germany : Lambert Academic Publishing, 2016.

[3] Niu Jinqi, Hongxing Cao, Baoshan Niu and Yongping Wu, "Study of atmospheric predictability based on periphery theory," Meteorology \& Environment Research, vol.6. no. 3, pp1-4, 2015.

[4] Yan Pengcheng, Wei Hou, Hongxing Cao et al. (2017, Mar, 11-12) "Research on system control based on a novel theory," Asia-Pacific Computer Science and Application Conference, Shanghai, ID: CSAC6518.

[5] Stocker, T. et al., "In: The physical Basis: Contribution of Working Group I to the Fifth Assessment Report of the Inter-government Panel on Climate Change" . IPCC Climate Change 2013, Cambridge, UK: Cambridge University Press, 2014.

[6] Peixoto J. P. and A. H. Oort, Physics of Climate, New York: Melville AIP Press, 1992.

[7] Shah, A.(2015, Feb) Climate Change and Global Warming. Global Issues. Available: http://www.globalissues.org/issue/178/climatechange-and-global-warming. 